\section{CONTEXT-DEPENDENT REVERSIBLE MODULATION OF CJUN EXPRESSION BY CAR T CELLS FOR CANCER TREATMENT}

'Zhifen Yang*, ${ }^{2}$ Francesco Marincola. ${ }^{1}$ Refuge Biotech, Menlo Park, CA, USA; ${ }^{2}$ Refuge Biotech, currently Gilead/Kite, Santa Monica, CA, USA

Background Overexpression of canonical AP-1 factor cJUN was shown to prevent CAR $\mathrm{T}$ cell exhaustion and improve anti-tumor potency in vivo (1). However, its clinical utilization is limited by potential for transformation and oncogenic risk. Here, we present a conditional, antigen-dependent, non-editing CRISPR-activation (CRISPRa) circuit (RB-339) that delivers context-dependent upregulation of endogenous cJUN increasing CAR-T cell resistance to exhaustion.

Methods RB-339 is a CAR T cell engineered to conditionally turn on the transcription of the cJUN endogenous gene. The circuit includes a lentiviral construct encoding an anti-HER2 (4D5) single chain variable fragment, with CD28 and CD3 $\zeta$ co-stimulatory domains linked to a tobacco etch virus (TEV) protease and a single guide RNA (sgRNA) targeting the promoter region of cJUN. A second construct encodes linker for activation of $\mathrm{T}$ cells, complexed to nuclease-deactivated/dead Cas9 (dCas9)-VP64-p65-Rta transcriptional activator (VPR) via a TEV-cleavable linker (LdCV). Activation of CAR allows the release of dCas9 for nuclear localization and conditionally and reversibly induces the expression of cJUN. RB-339 was compared in vitro to control (cRB-339, lacking the cJUN sgRNA) and CAR-T cells engineered to constitutively express cJun.

Results RB-339 induced cJUN upregulation upon stimulation with HER2-coated beads and this resulted in significantly elevated expression over a 6-day time course compared to the control cRB-339 (figure 1A-B). When HER2-coated beads were removed at day 3, cJUN expression returned to baseline parallel to cRB-339. The conditional upregulation of cJUN in RB-339 contrasted with the constitutive overexpression in the transgene carrying cells that was irrespective of antigen stimulation (figure 1C). Upon exposure to HER2 + FaDu cancer cells, RB-339 peaked at day 2 and declined afterwards when $\mathrm{FaDu}$ cells were killed at day 3; cJUN increased again at day 4 upon restimulation with $\mathrm{FaDu}$ cells at day 3 (figure 2). Such a dynamic induction of cJUN resulted in significantly enhanced CAR-T cells proliferation in RB-339 compared to the respective conventional CAR-T cells or cRB-339 (figure 3).

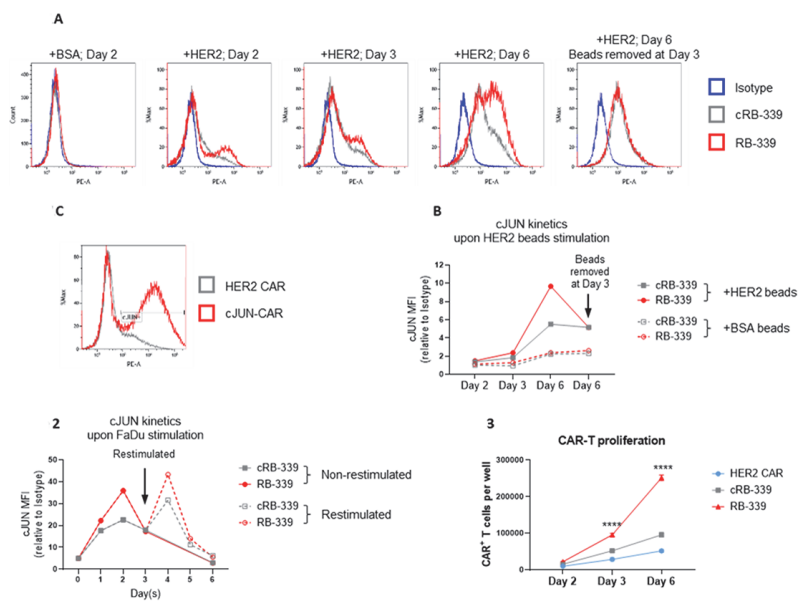

Abstract 154 Figure 1 Conditional upregulation of CJUN by RB-339 in vitro. RB-339 and its control CRB-339 were stimulated by HER2coated or BSA-coated beads for either six days or three days followed by removal of beads at day 3 (figure $1 \mathrm{~A}-\mathrm{B}$ ). Intracellular expression of cJUN was detected at indicated time points. Intracellular cJUN expression in overexpressed cJUN-CAR (figure 1C)

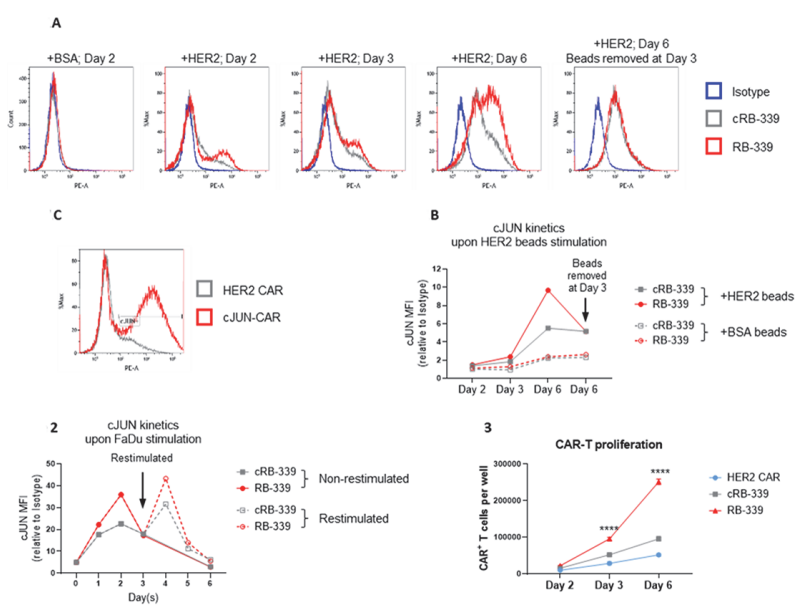

Abstract 154 Figure 2 Kinetics of cJUN upregulation in RB-339 upon exposure to HER2 + FaDu tumor cells. RB-339 and its control CRB-339 were stimulated by FaDu tumor cells for six days with or without restimulation at day 3

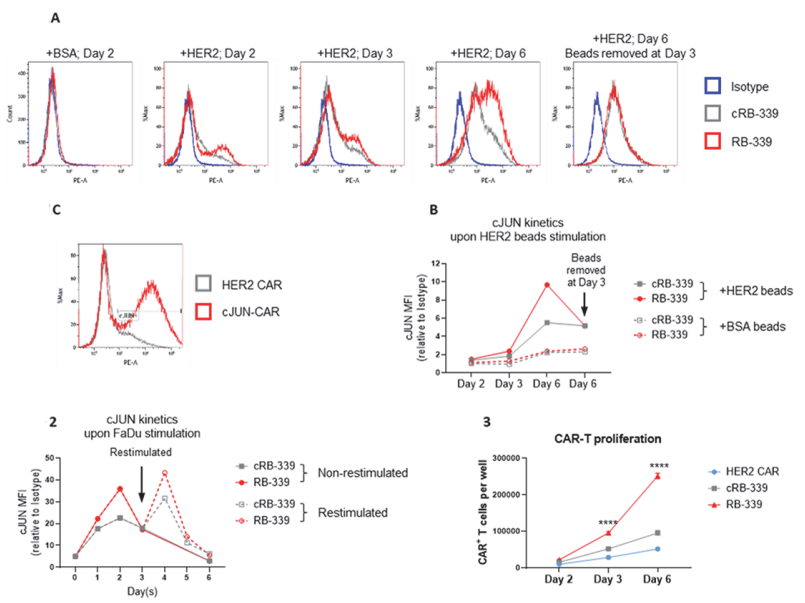

Abstract 154 Figure 3 Conditional upregulation of cJUN resulted in enhanced CAR-T proliferation in RB-339 in vitro after 6-day co-culture with FaDu tumor cells, compared to conventional HER2 CAR or CRB-339

Conclusions We conclude that CAR-T engineered to conditionally express the canonical AP-1 factor cJUN increases expansion potential similarly to CAR-T cells engineered to constitutively express the cJun transgene. However, the context-dependent upregulation of cJUN limits the risk of oncogenic transformation. We are currently combining inducible and reversible cJUN and IL-12 upregulation for the generation of the next RB-339 product.

\section{REFERENCE}

1. Lynn RC, Weber EW, et al. c-Jun overexpression in CAR T cells induces exhaustion resistance. Nature 2019; 576(7786):293-300.

http://dx.doi.org/10.1136/jitc-2021-SITC2021.154 